\title{
Resistência Quantitativa à Ferrugem da Folha em Genótipos de Aveia Branca: I - Caracterização da Reação em Condições de Campo*
}

\author{
Márcia S. Chaves ${ }^{1}$, José A. Martinelli² \& Luiz C. Federizzi ${ }^{3}$ \\ 1Embrapa Trigo, Cx. Postal 451, CEP 99001-970, Passo Fundo, RS, e-mail: mchaves@cnpt.embrapa.br; ${ }^{2}$ Departamento de \\ Fitossanidade, ${ }^{3}$ Departamento de Plantas de Lavoura, Faculdade de Agronomia, Universidade Federal do Rio Grande do Sul, \\ Cx. Postal 776, CEP 91540-000, Porto Alegre, RS
}

(Aceito para publicação em 26/09/2003)

Autor para correspondência: Márcia Soares Chaves

CHAVES, M.S., MARTINELLI, J.A. \& FEDERIZZI, L.C. Resistência quantitativa à ferrugem da folha em genótipos de aveia branca: I - Caracterização da reação em condições de campo. Fitopatologia Brasileira 29:039-046. 2004.

\section{RESUMO}

Entre as doenças que se manifestam na cultura da aveia (Avena sativa), a ferrugem da folha, causada por Puccinia coronata f. sp. avenae, tem-se mostrado a mais destrutiva, sendo responsável pelo decréscimo na qualidade e no rendimento dos grãos. O controle da doença através do uso de cultivares com resistência qualitativa vem sendo restringido pela capacidade do patógeno em superar este tipo de resistência. Visando possibilitar a utilização de uma estratégia alternativa para o controle da doença, foi investigada a ocorrência de resistência quantitativa em 31 genótipos de aveia branca. Os ensaios foram realizados durante os anos de 1996 a 2000, na Estação Experimental Agronômica da UFRGS, em Eldorado do Sul, RS, Brasil. Foi avaliado o progresso da doença no campo nos genótipos durante três anos, sendo que alguns deles foram testados durante cinco anos.
Para tal, foram realizadas avaliações semanais da severidade da ferrugem nas parcelas, traçando-se, a partir destes dados, as curvas de progresso da doença, sendo calculadas também as áreas delimitadas por essas curvas (AACPD) e a taxa de progresso da doença $(r)$. Em todos os anos houve grande variabilidade entre os genótipos quanto à reação à ferrugem da folha, e, conforme sua reação ao longo dos anos em que foram testados, os genótipos foram classificados em quatro grupos: resistentes, moderadamente resistentes, moderadamente suscetíveis e suscetíveis. Os dois primeiros grupos apresentam bons níveis de resistência quantitativa e poderão ser usados futuramente como genitores em programas de melhoramento genético.

Palavras-chaves adicionais: Puccinia coronata f. sp. avenae, Avena sativa, seleção fenotípica, AACPD.

\section{ABSTRACT}

Quantitative resistance to crown rust in white oat genotypes: I - Characterization of reaction in field conditions

Among diseases that attack oat (Avena sativa) crop, crown rust, caused by Puccinia coronata f. sp. avenae, has been the most destructive, being responsible for a decrease in the quality and yield of grains. Control through qualitative resistance has been restricted by the capacity of the pathogen to overcome this resistance type. Seeking an alternative strategy for disease control, 31 white oat genotypes were investigated for the occurrence of quantitative resistance. Trials were accomplished from 1996 to 2000, in the Agronomic Experimental Station of UFRGS, in Eldorado do Sul, RS,
Brazil. Disease progress was evaluated in the field for three years, and some genotypes were tested for five years. Weekly evaluations of rust severity in each plot were performed, providing data from which to determine the disease progress curves, and calculate the area under the disease progress curve (AUDPC), and the rate of development of disease $(r)$. There was great variability among genotypes for all the years in regard to rust reaction, allowing the genotypes to be classified in four groups: resistant, moderately resistant, moderately susceptible and susceptible. The first two groups have good levels of quantitative resistance and can be used in the future as parents in breeding programs.

\section{INTRODUÇÃO}

A ferrugem da folha, causada por Puccinia coronata $\mathrm{f}$. sp. avenae Fraser. \& Led., é a doença mais danosa dentre aquelas que afetam a aveia branca (Avena sativa L.), tendo causado severas epidemias em todas as regiões do mundo onde este cereal é cultivado. $\mathrm{O}$ dano causado às folhas, especialmente à folha bandeira, ocasionando a redução da

\footnotetext{
* Parte da Tese de Doutorado do primeiro autor. Universidade Federal do Rio Grande do S4ul (2001).

${ }^{2,3}$ Bolsista do $\mathrm{CNPq}$
}

fotossíntese, interfere na redistribuição dos produtos resultantes deste processo das folhas para o grão em formação. Isto resulta em grãos murchos, com pouco ou nenhum valor comercial e nutricional (Simons, 1985). As características ligadas ao rendimento mais afetadas pela ocorrência da doença são o peso médio de panículas e o peso de 1.000 sementes (Cruz et al, 1999). O rendimento e a qualidade de grãos podem sofrer decréscimos acima de $30 \%$, podendo chegar a $50 \%$ em cultivares suscetíveis, dependendo do nível de incidência da doença (Simons et al, 1983; Martinelli et al, 1994). Doehlert \& McMullen (2000) observaram altas taxas de quebra dos grãos 
durante o descascamento, em lotes de aveia oriundos de locais onde ocorreram epidemias severas da ferrugem da folha.

O uso de cultivares resistentes é considerado o método de controle mais efetivo e econômico para as ferrugens dos cereais (Priestley \& Bayles, 1988; Harder \& Haber, 1992). Tradicionalmente, em programas de melhoramento genético tem sido utilizada a resistência qualitativa, condicionada por genes de efeitos maiores, em virtude da relativa facilidade de sua transferência a genótipos elite. Entretanto, este tipo de resistência, com algumas exceções, não é durável por longos períodos quando usada em grandes áreas (Parlevliet, 1988). Isto ocorre porque as ferrugens, sendo parasitas obrigatórios, co-evoluíram com seus hospedeiros como componentes de um sistema muito influenciado pelas condições ecológicas, ou seja, qualquer mudança na população predominante do hospedeiro, resulta em mudanças subsequentes na população do patógeno, para que o equilíbrio seja reestabelecido (Wahl et al., 1984).

No sul do Brasil existem vários fatores que, juntamente com características intrínsecas ao patógeno, contribuem para que haja uma pressão de seleção muito grande sobre a população de $P$. coronata f. sp. avenae, fazendo com que os genes de resistência qualitativa sejam efetivos durante poucos anos (Zimmer et al., 1963; Federizzi \& Stuthman, 1998).

A piramidização de genes maiores de resistência no hospedeiro tem sido sugerida por alguns pesquisadores para o aumento da vida útil das cultivares (Nelson, 1978). Contudo, em um estudo recente, Martinelli et al. (1998) observaram que a estrutura da população de $P$. coronata $\mathrm{f}$. sp. avenae no sul do Brasil é extremamente diversificada e que as raças predominantes possuem de dez a 26 genes de virulência por raça. O espectro de virulência das raças encontradas no sul do Brasil é semelhante ou superior ao de outros países nos quais este monitoramento é realizado, incluindo Israel, considerado o centro de origem do patógeno, e os EUA, nos quais o ciclo do fungo é completo (Leonard, 1999). Esta população parece ser uma das mais complexas do mundo em função da alta variabilidade das raças que a constituem e da diversidade de suas virulências. Neste caso, a piramidização de genes, deve ser cuidadosamente avaliada, pois os dados sugerem que sua utilização pode não alcançar os resultados esperados.

Face à esta situação, torna-se necessário estudar outras formas de resistência, as quais possam ser utilizadas como novas estratégias, na busca de uma maior estabilidade da cultura da aveia frente às epidemias de ferrugem da folha. Nesse contexto, o objetivo deste trabalho foi avaliar a reação de genótipos de aveia, visando identificar aqueles com potencial resistência quantitativa à doença. Este tipo de resistência tem sido relatado em várias interações patógeno-hospedeiro, dentre as quais encontram-se algumas ferrugens em cereais (Luke et al., 1975; Johnson \& Wilcoxson, 1978; Zhang, 1995; Alemayehu \& Parlevliet, 1996; Pataky et al., 1998) e sua principal característica observada a campo é o progresso lento da doença (Parlevliet, 1979; 1985). Existem evidências de que a resistência quantitativa pode apresentar maior durabilidade que a qualitativa, quando utilizada em grandes áreas durante um longo período de tempo (Wilcoxson, 1981; Johnson, 1984).

\section{MATERIALEMÉTODOS}

\section{Genótipos de aveia}

Foram avaliados 31 genótipos de aveia branca (linhagens do Programa de Melhoramento Genético de Aveia da Faculdade de Agronomia da UFRGS e cultivares) apresentando diferentes reações à ferrugem da folha da aveia. O conjunto de genótipos foi testado durante os anos de 1996 a 2000 (Tabela 1).

\section{Ensaios}

Os ensaios foram conduzidos em condições de campo, na Estação Experimental Agronômica da Universidade Federal do Rio Grande do Sul (EEA/UFRGS), km 146 da rodovia BR290, município de Eldorado do Sul, localizado na região ecofisiográfica da Depressão Central. As coordenadas são $30^{\circ} 05$ ' $22^{\prime}$ ' $\mathrm{S}$ de latitude e $51^{\circ} 39^{\prime} 08^{\prime \prime} \mathrm{W}$ de longitude, com altitude de aproximadamente $46 \mathrm{~m}$. O clima neste local é subtropical úmido de verão quente, variedade específica "Cfa". O solo pertence à Unidade de Mapeamento Arroio dos Ratos, classificado como laterítico hidromórfico (Mello et al., 1996). De acordo com o sistema brasileiro de classificação, caracterizase como um plintossolo (Camargo et al., 1987).

O sistema de plantio direto com semeadura mecânica foi adotado, utilizando-se uma semeadora de parcelas experimentais. As datas de semeadura foram 4 de julho de 1996, 11 de junho de 1997, 16 de junho de 1998, 8 de julho de 1999 e 12 de junho de 2000. Nos estádios de duas e cinco folhas, foram realizadas aplicações de nitrogênio em cobertura, com doses de $40 \mathrm{~kg}$ de $\mathrm{N} / \mathrm{ha}$. O delineamento experimental foi de blocos ao acaso com três repetições, tendo cada parcela cinco linhas de $3 \mathrm{~m}$, espaçadas entre si por $0,2 \mathrm{~m}$. Os ensaios tiveram inoculação natural.

\section{Avaliações}

A resistência parcial deve ser avaliada no campo, através da quantificação da proporção do tecido do hospedeiro afetado. Esta medição pode ser realizada uma vez, próximo ao final do ciclo da moléstia, ou várias vezes durante o desenvolvimento da epidemia. A primeira medição representa o resultado acumulado do efeito dos componentes de resistência ao longo do tempo, e a segunda possibilita o cálculo da área abaixo da curva de progresso da doença (AACPD) e da taxa aparente de infecção $(r)$ (Parlevliet, 1988). Das três formas de avaliação citadas, a última ( $r$ ) é considerada como claramente inferior às outras duas (severidade final e AACPD) (Rees et al., 1979a; Rees et al,. 1979b; Parlevliet, 1985). Para quantificar a resistência dos genótipos testados foram feitas avaliações semanais da severidade da doença em cada parcela, desde o surgimento das primeiras pústulas até próximo ao final do ciclo, com o auxílio de uma escala diagramática (Stubbs et al., 1986). Em 1996 e 2000 foram realizadas sete avaliações; em 1997 foram seis avaliações e em 1998 e 1999, oito avaliações. A partir destes dados foram traçadas as curvas de progresso da doença para 
Resistência quantitativa à ferrugem da folha em genótipos de aveia...

TABELA 1 - Genótipos de aveia (Avena sativa) e respectivas genealogias, utilizados para o estudo da resistência quantitativa à ferrugem da folha em condições de campo (EEA/UFRGS, Eldorado do Sul, RS), nos anos de 1996 a 2000

\begin{tabular}{|c|c|c|c|c|}
\hline \multirow{2}{*}{ Genótipo } & \multirow{2}{*}{ Genealogia } & \multicolumn{3}{|c|}{ Anos } \\
\hline & & 1996 & 1997 & 1998 a 2000 \\
\hline UFRGS 41698-22 & UFRGS 881920/ UFRGS 7 & $\mathrm{x}$ & $\mathrm{x}$ & $\mathrm{x}$ \\
\hline UFRGS 14 & UFRGS $805165 / / \mathrm{COR}^{2} / \mathrm{CTZ}^{3} /$ Pendek/Me 1563 & $\mathrm{x}$ & $\mathrm{x}$ & $\mathrm{x}$ \\
\hline UFRGS 42085-10 & UFRGS 881920/ UFRGS 14 & $\mathrm{x}$ & $\mathrm{x}$ & $\mathrm{x}$ \\
\hline UFRGS 17 & $\mathrm{COR}^{2} / \mathrm{CTZ}^{3} /$ Pendek/Me 1563//76-29/76-23/75-88/CI 833 & $\mathrm{x}$ & $\mathrm{x}$ & $\mathrm{x}$ \\
\hline UFRGS 41709-5 & UFRGS 15/ UFRGS 881920 & $\mathrm{x}$ & $\mathrm{x}$ & $\mathrm{x}$ \\
\hline UFRGS 10 & CI 24//Coronado/BCLA & $\mathrm{x}$ & $\mathrm{x}$ & $\mathrm{x}$ \\
\hline UFRGS 41699-3 & UFRGS 10/ UFRGS 8 & $\mathrm{x}$ & $\mathrm{x}$ & $\mathrm{x}$ \\
\hline UFRGS 7 & X1205/FLA 1093 & $\mathrm{x}$ & $\mathrm{x}$ & $\mathrm{x}$ \\
\hline UFRGS 953167 & UFRGS 884087/ UFRGS 15 & $\mathrm{x}$ & $\mathrm{x}$ & $\mathrm{x}$ \\
\hline UFRGS 952403 & 1UFRGS 881920/ UFRGS 14 & & $\mathrm{x}$ & $\mathrm{x}$ \\
\hline UFRGS 953109 & UFRGS 15/UFRGS 10 & & $\mathrm{x}$ & $\mathrm{x}$ \\
\hline UFRGS 952602 & UFRGS 8/ UFRGS 881920 & & $\mathrm{x}$ & $\mathrm{x}$ \\
\hline UFRGS 94638-B & UFRGS 884110/UFRGS 884020 & $\mathrm{x}$ & & $\mathrm{x}$ \\
\hline UFRGS 940772 & UFRGS 881920/ UFRGS 15 & $\mathrm{x}$ & & $\mathrm{x}$ \\
\hline UFRGS 41721-21 & UFRGS 884087/UFRGS 15 & $\mathrm{x}$ & & $\mathrm{x}$ \\
\hline UFRGS 911748-1 & NC 2469-3/CURT//UFRGS 15 & $\mathrm{x}$ & & $\mathrm{x}$ \\
\hline UFRGS 953133 & UFRGS 15/UFRGS 881920 & & & $\mathrm{x}$ \\
\hline UFRGS 953304 & UFRGS881920/UFRGS 15 & & & $\mathrm{x}$ \\
\hline UFRGS 953285 & UFRGS881920/UFRGS 15 & & & $\mathrm{x}$ \\
\hline UFRGS 16 & C 16 CRcpx/C 7512/SRcpx/74 C 8014 & & & $\mathrm{x}$ \\
\hline CTC 3 & Steele// $\mathrm{COR}^{2} / \mathrm{CTZ}^{3} /$ Pendek/Me 1563 & & & $\mathrm{x}$ \\
\hline UFRGS 940263-3 & UFRGS 19= UFRGS884110/UFRGS 884021-1 & & & $\mathrm{x}$ \\
\hline UFRGS 910906 & UFRGS 15/UFRGS 881920 & & & $\mathrm{x}$ \\
\hline UFRGS 922003 & Seleção UFRGS 898065 & & & $\mathrm{x}$ \\
\hline UFRGS 93576 & UFRGS10/UFRGS 8 & & & $\mathrm{x}$ \\
\hline UFRGS 93641 & UFRGS 884087/UFRGS 15 & & & $\mathrm{x}$ \\
\hline UFRGS 921260 & UFRGS 84087UFRGS 881920 & & & $\mathrm{x}$ \\
\hline UFRGS 950120 & & & & $\mathrm{x}$ \\
\hline UFRGS 952576-1/2 & UPF 850380/90 SAT-1 [Guaiba sel\#1] & & & $\mathrm{x}$ \\
\hline UFRGS $960282-1$ & UFRGS 881920/UFRGS 14 & & & $\mathrm{x}$ \\
\hline UFRGS 960845-2 & UFRGS 884110/UFRGS 884021-1 & & & $\mathrm{x}$ \\
\hline
\end{tabular}

cada genótipo testado, e calculadas as áreas delimitadas por estas curvas através de integração trapezoidal, utilizando a equação:

$$
\mathrm{AACPD}=\sum_{i=1}^{n}\left(\left[\left(\mathrm{Y}_{i+1}+\mathrm{Y}_{i}\right) \mathrm{x} 0,5\right]\left[\mathrm{T}_{i+1}-\mathrm{T}_{i}\right]\right.
$$

onde $\mathrm{Y}_{i}=$ percentagem de área foliar afetada pela ferrugem na $i$-ésima observação, $\mathrm{T}_{i}=$ tempo (em dias) no momento da $i$ ésima observação e $n=$ número total de observações (Shaner \& Finney, 1977). Com os dados coletados foram também estimadas as taxas de progresso da doença ( $r=$ percentagem de área foliar afetada/dia).

\section{Análise estatística}

A análise estatística dos dados foi feita através do programa SANEST-Sistema de Análise Estatística para Microcomputadores (Zonta \& Machado, 1984). Os dados de área abaixo da curva de progresso da doença (AACPD), severidade final e taxa de progresso de doença foram submetidos à análise de variância (ANOVA), e a discriminação da variação entre genótipos foi feita pelo teste de Tukey ao nível de 5\% de probabilidade. Os dados de AACPD sofreram transformação do tipo raiz quadrada de $(x+10)$, e os dados de severidade final e taxa de progresso, transformação do tipo arco seno da raiz quadrada de (x/100).

\section{RESULTADOS}

Houve diferenças significativas entre os genótipos testados quanto a AACPD, severidade final e taxa de progresso da doença nos cinco anos de avaliações $(\mathrm{P}>\mathrm{F}=0,00001)$.

Em 1996, a ferrugem da folha ocorreu durante aproximadamente 52 dias (do início de setembro até o final de outubro). Os valores de AACPD variaram entre 2,1 e 502,1 (Tabela 2). A severidade final variou de $0,1 \%$ a $63,5 \%$ (Tabela 3 ), observando-se valores de taxa de progresso da ferrugem entre $0,002 \% /$ dia e $2,48 \% /$ dia (Tabela 4 ). Os genótipos mais 
M.S. Chaves et al.

suscetíveis neste ano foram UFRGS 7, UFRGS 14 e UFRGS 10. Dentre os mais resistentes destacaram-se os genótipos UFRGS 41721-21 e UFRGS 94638-B.

Em 1997, a doença foi observada durante um período aproximado de 63 dias (do final de agosto até o início de novembro), tendo sido verificados valores de AACPD que variaram entre 48,4 e 1126 (Tabela 2). A severidade final variou de $2,5 \%$ a $53,3 \%$ (Tabela 3 ), observando-se valores de taxa de progresso da ferrugem entre $0,121 \% /$ dia e $3,277 \%$ /dia (Tabela 4). Os genótipos UFRGS 7, UFRGS 14 e UFRGS 10 tiveram novamente alta suscetibilidade neste ano. Dentre os mais resistentes destacaram-se os genótipos UFRGS 952403, UFRGS 953109 e UFRGS 41698-22.

Em 1998, a doença ocorreu durante aproximadamente 68 dias (da metade de agosto até o final de outubro). Os valores de AACPD variaram entre 21,5 e 2654 (Tabela 2). A severidade final variou de $0,7 \%$ a $100 \%$ (Tabela 3 ), observando-se valores de taxa de progresso da ferrugem entre $0,004 \%$ /dia e 1,626\%/ dia (Tabela 4). Os genótipos UFRGS 14 e UFRGS 952403 se destacaram em suscetibilidade neste ano. Dentre os mais resistentes destacaram-se os genótipos UFRGS 960845-2, UFRGS 910906, UFRGS 16 e CTC 3.

Em 1999, a ferrugem foi observada durante cerca de 49 dias (da metade de setembro até o final de outubro). Os valores de AACPD variaram entre 10,9 e 196,7 (Tabela 2). A severidade final variou de $1,1 \%$ a $43,3 \%$ (Tabela 3 ), observando-se valores de taxa de progresso da ferrugem entre $0,026 \%$ /dia e $1,326 \%$ / dia (Tabela 4). O genótipo UFRGS 7 se destacou dos demais, sendo o mais suscetível. Tiveram também alta suscetibilidade neste ano UFRGS 14 e UFRGS 952576-1/2. Dentre os mais resistentes destacaram-se os genótipos UFRGS 960845-2, UFRGS 952403 e UFRGS 953285.

Em 2000, a doença ocorreu durante cerca de 57 dias (do início de setembro até o final de outubro), sendo que os valores

TABELA 2 - Área abaixo da curva de progresso da doença (AACPD) em genótipos de aveia branca (Avena sativa), em condições de campo (EEA/UFRGS, Eldorado do Sul, RS) nos anos de 1996 a 2000

\begin{tabular}{|c|c|c|c|c|c|}
\hline \multirow{2}{*}{ Genótipo } & \multicolumn{5}{|c|}{ AACPD } \\
\hline & 1996 & 1997 & 1998 & 1999 & 2000 \\
\hline UFRGS 7 & $502,1 \mathrm{a}$ & $1126,0 \mathrm{a}$ & 2204,8 abcd & 196,7 a & 756,4 abcd \\
\hline UFRGS 10 & $100,6 \mathrm{bc}$ & $579,7 \mathrm{ab}$ & $2220,8 \mathrm{abc}$ & $159,2 \mathrm{ab}$ & 597,5 abcdef \\
\hline UFRGS 14 & $153,2 \mathrm{~b}$ & $434,6 a b c$ & $2654,0 \mathrm{a}$ & 119,6 abcd & 798,3 abc \\
\hline UFRGS 17 & $15,8 \mathrm{e}$ & 262,9 bcde & 1870,0 cdefg & 115,64 abcd & 554,7 bcdef \\
\hline UFRGS 41699-3 & $88,1 \mathrm{bc}$ & $114,0 \mathrm{def}$ & 1832,3 cdefg & 47,6 abcdefg & 531,5 bcdefg \\
\hline UFRGS 953109 & -- & 69,2 ef & 1616,9 efghij & 42,5 bcdefg & 589,7 abcdef \\
\hline UFRGS 952403 & --- & $48,4 \mathrm{f}$ & $2375,7 \mathrm{ab}$ & $22,8 \mathrm{efg}$ & 506,3 bcdefg \\
\hline UFRGS 952602 & --- & 242,9 bcde & 1306,1 hijklm & 118,1 abcd & 664,9 abcdef \\
\hline UFRGS 922003 & --- & --- & 2010,7 bcdef & 99,0 abcde & 661,3 abcdef \\
\hline UFRGS 921260 & $15,0 \mathrm{e}$ & --- & 1622,3 efghij & 36,7 bcdefg & 524,9 bcdefg \\
\hline UFRGS 950120 & -- & --- & 1747,8 defgh & 117,96 abcd & 947,3 a \\
\hline UFRGS 952576-1/2 & --- & --- & $2222,3 \mathrm{abc}$ & $147,3 \mathrm{abc}$ & $876,8 \mathrm{ab}$ \\
\hline UFRGS 960286-1 & -- & --- & 1975,3 bcdef & 96,5 abcde & 467,9 cdefg \\
\hline UFRGS 953167 & -- & $154,3 \mathrm{cdef}$ & 1562,3 fghijk & 95,7 abcde & 611,5 abcdef \\
\hline UFRGS 940263-3 & --- & --- & $915,7 \mathrm{n}$ & 53,1 abcdefg & 688,5 abcde \\
\hline UFRGS 94638-B & $2,1 \mathrm{e}$ & --- & 1199,9 jklmn & 82,85 abcde & 586,6 bcdef \\
\hline UFRGS 940772 & $16,5 \mathrm{e}$ & --- & $1131,7 \mathrm{klmn}$ & 39,2 bcdefg & 584,9 abcdef \\
\hline UFRGS 953285 & --- & --- & 1305,1 hijklmn & $13,0 \mathrm{fg}$ & 491,8 cdefg \\
\hline CTC 3 & --- & --- & $1057,4 \mathrm{lmn}$ & 45,0 bcdf $\mathrm{g}$ & 472,3 cdefg \\
\hline UFRGS 911748-1 & $9,6 \mathrm{e}$ & --- & 1268,4 ijklmn & 72,5 abcdef & 611,2 abcdef \\
\hline UFRGS 41698-22 & $18,8 \mathrm{de}$ & 99,8 ef & $1129,7 \mathrm{klmn}$ & 47,92 abcdefg & 348,3 efgh \\
\hline UFRGS 41709-5 & $13,9 \mathrm{e}$ & 408,0 abcd & 1693,9 efghi & 118,1 abcd & 424,7 defgh \\
\hline UFRGS 42085-10 & $54,5 \mathrm{~cd}$ & 262,4 bcde & 2084,9 bcde & 51,5 abcdefg & 423,5 defgh \\
\hline UFRGS 41721-21 & $13,8 \mathrm{e}$ & --- & 1406,6 ghijklm & 70,3 abcdef & 472,3 cdefg \\
\hline UFRGS 953133 & -- & --- & $1141,4 \mathrm{klmn}$ & 26,9 defg & $312,2 \mathrm{fgh}$ \\
\hline UFRGS 16 & --- & --- & $973,6 \mathrm{mn}$ & 31,7 defg & 359,1 efgh \\
\hline UFRGS 93641 & --- & --- & $1118,9 \mathrm{klmn}$ & 26,2 defg & 425,9 cdefgh \\
\hline UFRGS 93576 & -- & --- & $1176,7 \mathrm{jklmn}$ & 40,2 bcdefg & $304,8 \mathrm{fgh}$ \\
\hline UFRGS 953304 & --- & --- & 1496,4 ghijkl & 33,6 cdefg & 344,0 efgh \\
\hline UFRGS 910906 & --- & --- & $989,6 \mathrm{mn}$ & 26,3 defg & $171,9 \mathrm{gh}$ \\
\hline UFRGS 960845-2 & --- & --- & 21,15 o & $10,9 \mathrm{G}$ & $85,1 \mathrm{~h}$ \\
\hline C. V. (\%) & 7,73 & 7,81 & 9,56 & 9,55 & 22,15 \\
\hline
\end{tabular}

Os dados sofreram transformação do tipo raiz quadrada de $(x+10)$.

Médias seguidas por letras distintas diferem entre si pelo teste de Tukey ao nível de 5\% de probabilidade. 
Resistência quantitativa à ferrugem da folha em genótipos de aveia...

TABELA 3 - Severidade final de ferrugem da folha em genótipos de aveia branca (Avena sativa), em condições de campo (EEA/UFRGS, Eldorado do Sul, RS) nos anos de 1996 a 2000

\begin{tabular}{|c|c|c|c|c|c|c|c|c|c|}
\hline \multirow{3}{*}{$\begin{array}{l}\text { Genótipo } \\
\text { UFRGS } 7\end{array}$} & \multicolumn{9}{|c|}{$r$ (\%Área foliar afetada/dia) } \\
\hline & 1996 & \multicolumn{2}{|c|}{1997} & \multicolumn{2}{|c|}{1998} & \multicolumn{2}{|c|}{1999} & \multicolumn{2}{|c|}{2000} \\
\hline & 2,480 a & 3,277 & $\mathrm{a}$ & 1,507 & abcd & 1,326 & a & 2,043 & abc \\
\hline UFRGS 10 & $0,661 \mathrm{~b}$ & 1,855 & $\mathrm{ab}$ & 1,476 & abcde & 0,607 & abcde & 1,857 & abcd \\
\hline UFRGS 14 & $0,729 \mathrm{~b}$ & 1,894 & $a b$ & 1,627 & a & 0,928 & $\mathrm{ab}$ & 2,048 & $a b c$ \\
\hline UFRGS 17 & $0,050 \mathrm{~cd}$ & 0,954 & bcd & 1,408 & bcdefgh & 0,912 & $a b c$ & 1,762 & abcde \\
\hline UFRGS 41699-3 & $0,334 \mathrm{bc}$ & 0,247 & cd & 1,489 & abcde & 0,475 & bcdef & 1,447 & bcdefgh \\
\hline UFRGS 953109 & -- & 0,135 & $\mathrm{~cd}$ & 1,425 & bcdefg & 0,499 & bcdef & 2,029 & $\mathrm{abc}$ \\
\hline UFRGS 952403 & --- & 0,121 & d & 1,549 & $a b c$ & 0,178 & efg & 1,729 & abcde \\
\hline UFRGS 952602 & --- & 0,856 & bed & 1,426 & bcdefg & 0,495 & bcdef & 1,979 & abc \\
\hline UFRGS 922003 & --- & -- & & 1,473 & abcde & 0,716 & abcd & 1,790 & abcd \\
\hline UFRGS 921260 & $0,063 \mathrm{~cd}$ & --- & & 1,401 & bcdefgh & 0,285 & cdefg & 1,549 & abcdefgh \\
\hline UFRGS 950120 & --- & -- & & 1,509 & abcd & 0,666 & abcde & 2,151 & $\mathrm{ab}$ \\
\hline UFRGS 952576-1/2 & --- & --- & & 1,492 & abcde & 0,914 & $\mathrm{ab}$ & 2,176 & $\mathrm{a}$ \\
\hline UFRGS 960286-1 & --- & --- & & 1,468 & abcde & 0,456 & bcdef & 1,585 & abcdefg \\
\hline UFRGS 953167 & --- & 0,493 & bcd & 1,454 & abcdef & 0,897 & $\mathrm{abc}$ & 1,939 & $\mathrm{abc}$ \\
\hline UFRGS 940263-3 & --- & -- & & 1,288 & ghij & 0,407 & bcdef & 1,871 & abcd \\
\hline UFRGS 94638-B & $0,002 \mathrm{~d}$ & --- & & 1,332 & efghi & 0,653 & abcde & 1,863 & abcd \\
\hline UFRGS 940772 & $0,063 \mathrm{~cd}$ & --- & & 1,211 & $\mathrm{ijkl}$ & 0,346 & bcdef & 1,766 & abcd \\
\hline UFRGS 953285 & -- & --- & & 1,297 & fghi & 0,092 & $\mathrm{fg}$ & 1,688 & abcdef \\
\hline CTC 3 & --- & --- & & 1,072 & 1 & 0,306 & bcdefg & 1,646 & abcdefg \\
\hline UFRGS 911748-1 & $0,023 \mathrm{~cd}$ & --- & & 1,302 & fghi & 0,532 & bcde & 1,687 & abcdef \\
\hline UFRGS 41698-22 & $0,101 \mathrm{~cd}$ & 0,221 & cd & 1,150 & $\mathrm{jkl}$ & 0,308 & bcdefg & 1,244 & defgh \\
\hline UFRGS 41709-5 & $0,065 \mathrm{~cd}$ & 1,363 & $a b c$ & 1,378 & defgh & 0,507 & bcdef & 1,347 & cdefgh \\
\hline UFRGS 42085-10 & 0,229 bcd & 0,938 & bed & 1,571 & $a b$ & 0,378 & bcdef & 1,251 & defgh \\
\hline UFRGS 41721-21 & $0,015 \mathrm{~d}$ & -- & & 1,381 & cdefgh & 0,512 & bcdef & 1,366 & cdefg \\
\hline UFRGS 953133 & -- & --- & & 1,212 & ijkl & 0,221 & defg & 0,964 & $\mathrm{~h}$ \\
\hline UFRGS 16 & --- & --- & & 1,112 & & 0,295 & bcdefg & 1,114 & efgh \\
\hline UFRGS 93641 & --- & --- & & 1,262 & ghijk & 0,159 & efg & 1,416 & cdefgh \\
\hline UFRGS 93576 & -- & --- & & 1,258 & hijk & 0,232 & defg & 1,036 & $\mathrm{gh}$ \\
\hline UFRGS 953304 & --- & --- & & 1,377 & defgh & 0,273 & defg & 1,082 & fgh \\
\hline UFRGS 910906 & --- & --- & & 1,204 & ijkl & 0,179 & efg & 0,394 & $\mathrm{i}$ \\
\hline UFRGS 960845-2 & --- & -- & & 0,005 & $\mathrm{~m}$ & 0,026 & $\mathrm{~g}$ & 0,313 & $\mathrm{i}$ \\
\hline C. V. $(\%)$ & 31,76 & 29,96 & & 1,9 & & 19,2 & & 6,9 & \\
\hline
\end{tabular}

Os dados sofreram transformação do tipo arco seno da raiz quadrada de (x/100).

Médias seguidas por letras distintas diferem entre si pelo teste de Tukey ao nível de 5\% de probabilidade.

de AACPD variaram entre 85,1 e 947,3 (Tabela 2). A severidade final variou de $15 \%$ a $99,1 \%$ (Tabela 3 ), observando-se valores de taxa de progresso da ferrugem entre 0,313\%/dia e 2,177\%/ dia (Tabela 4). Os genótipos UFRGS 950120, UFRGS 952576-1/ 2, UFRGS 7 e UFRGS tiveram alta suscetibilidade neste ano. Dentre os mais resistentes destacaram-se os genótipos UFRGS 960845-2, UFRGS 910906, UFRGS 93576 e UFRGS 953133.

\section{DISCUSSÃO}

A resistência parcial ou quantitativa é um tipo de resistência incompleta, geralmente condicionada por genes menores, em que, embora o hospedeiro apresente reação de suscetibilidade, verifica-se uma baixa taxa de progresso da doença. Para que a resistência parcial possa ser identificada e caracterizada em um grupo de genótipos, é necessário que entre estes haja variabilidade para os diversos componentes que a condicionam. A grande variabilidade encontrada entre os genótipos estudados na expressão da resistência em condições de campo, indica a possibilidade de seleção de constituições genéticas que apresentem a característica.

Analisando-se as AACPDs do conjunto de genótipos em cada ano, pode-se verificar que em 1998 e 2000 a epidemia foi severa, observando-se altos valores desta variável. Em 1998, a doença teve início excepcionalmente mais cedo (em meados de agosto), sendo que este fato contribuiu para que neste ano ocorressem os altos valores de AACPD observados. Em 1997, observa-se valores elevados de AACPD em alguns genótipos mais suscetíveis e também valores baixos, em outros genótipos, considerando-se, portanto, que neste ano a epidemia teve intensidade moderada. Nos anos de 1996 e 1999 a doença ocorreu com baixa intensidade Estes dados refletem a influência do ambiente na expressão deste tipo de característica.

Dos fatores relacionados ao ambiente, a temperatura é 
M.S. Chaves et al.

TABELA 4 - Taxa de progresso $(r)$ da ferrugem da folha em genótipos de aveia branca (Avena sativa), em condições de campo (EEA/UFRGS, Eldorado do Sul, RS) nos anos de 1996 a 2000

\begin{tabular}{|c|c|c|c|c|c|c|c|c|c|c|}
\hline \multirow{3}{*}{$\begin{array}{l}\text { Genótipo } \\
\text { UFRGS } 7\end{array}$} & \multicolumn{10}{|c|}{ r $r$ (\%Área foliar afetada/dia) } \\
\hline & \multicolumn{2}{|c|}{1996} & \multicolumn{2}{|c|}{1997} & \multicolumn{2}{|c|}{1998} & \multicolumn{2}{|c|}{1999} & \multicolumn{2}{|c|}{2000} \\
\hline & 2,480 & $\mathrm{a}$ & 3,277 & $\mathrm{a}$ & 1,507 & abcd & 1,326 & $\mathrm{a}$ & 2,043 & $a b c$ \\
\hline UFRGS 10 & 0,661 & $\mathrm{~b}$ & 1,855 & $a b$ & 1,476 & abcde & 0,607 & abcde & 1,857 & abcd \\
\hline UFRGS 14 & 0,729 & $\mathrm{~b}$ & 1,894 & $\mathrm{ab}$ & 1,627 & a & 0,928 & $\mathrm{ab}$ & 2,048 & $a b c$ \\
\hline UFRGS 17 & 0,050 & $\mathrm{~cd}$ & 0,954 & bcd & 1,408 & bcdefgh & 0,912 & $a b c$ & 1,762 & abcde \\
\hline UFRGS 41699-3 & 0,334 & bc & 0,247 & $\mathrm{~cd}$ & 1,489 & abcde & 0,475 & bedef & 1,447 & bcdefgh \\
\hline UFRGS 953109 & --- & & 0,135 & $\mathrm{~cd}$ & 1,425 & bcdefg & 0,499 & bcdef & 2,029 & abc \\
\hline UFRGS 952403 & -- & & 0,121 & $\mathrm{~d}$ & 1,549 & $\mathrm{abc}$ & 0,178 & efg & 1,729 & abcde \\
\hline UFRGS 952602 & --- & & 0,856 & bcd & 1,426 & bcdefg & 0,495 & bcdef & 1,979 & $a b c$ \\
\hline UFRGS 922003 & --- & & -- & & 1,473 & abcde & 0,716 & abcd & 1,790 & abcd \\
\hline UFRGS 921260 & 0,063 & $\mathrm{~cd}$ & -- & & 1,401 & bcdefgh & 0,285 & cdefg & 1,549 & abcdefgh \\
\hline UFRGS 950120 & -- & & -- & & 1,509 & abcd & 0,666 & abcde & 2,151 & $a b$ \\
\hline UFRGS 952576-1/2 & --- & & --- & & 1,492 & abcde & 0,914 & $\mathrm{ab}$ & 2,176 & $\mathrm{a}$ \\
\hline UFRGS 960286-1 & --- & & --- & & 1,468 & abcde & 0,456 & bcdef & 1,585 & abcdefg \\
\hline UFRGS 953167 & --- & & 0,493 & bcd & 1,454 & abcdef & 0,897 & $\mathrm{abc}$ & 1,939 & $\mathrm{abc}$ \\
\hline UFRGS 940263-3 & --- & & -- & & 1,288 & ghij & 0,407 & bcdef & 1,871 & abcd \\
\hline UFRGS 94638-B & 0,002 & $\mathrm{~d}$ & --- & & 1,332 & efghi & 0,653 & abcde & 1,863 & abcd \\
\hline UFRGS 940772 & 0,063 & cd & -- & & 1,211 & ijkl & 0,346 & bcdef & 1,766 & abcd \\
\hline UFRGS 953285 & --- & & --- & & 1,297 & fghi & 0,092 & $\mathrm{fg}$ & 1,688 & abcdef \\
\hline CTC 3 & --- & & -- & & 1,072 & 1 & 0,306 & bcdefg & 1,646 & abcdefg \\
\hline UFRGS 911748-1 & 0,023 & $\mathrm{~cd}$ & -- & & 1,302 & fghi & 0,532 & bcde & 1,687 & abcdef \\
\hline UFRGS 41698-22 & 0,101 & $\mathrm{~cd}$ & 0,221 & $\mathrm{~cd}$ & 1,150 & $\mathrm{jkl}$ & 0,308 & bcdefg & 1,244 & defgh \\
\hline UFRGS 41709-5 & 0,065 & $\mathrm{~cd}$ & 1,363 & $a b c$ & 1,378 & defgh & 0,507 & bcdef & 1,347 & cdefgh \\
\hline UFRGS 42085-10 & 0,229 & bcd & 0,938 & bcd & 1,571 & $a b$ & 0,378 & bcdef & 1,251 & defgh \\
\hline UFRGS 41721-21 & 0,015 & $\mathrm{~d}$ & --- & & 1,381 & cdefgh & 0,512 & bcdef & 1,366 & cdefg \\
\hline UFRGS 953133 & -- & & -- & & 1,212 & $\mathrm{ijkl}$ & 0,221 & defg & 0,964 & $\mathrm{~h}$ \\
\hline UFRGS 16 & --- & & --- & & 1,112 & & 0,295 & bcdefg & 1,114 & efgh \\
\hline UFRGS 93641 & --- & & --- & & 1,262 & ghijk & 0,159 & efg & 1,416 & cdefgh \\
\hline UFRGS 93576 & --- & & -- & & 1,258 & hijk & 0,232 & defg & 1,036 & $\mathrm{gh}$ \\
\hline UFRGS 953304 & --- & & --- & & 1,377 & defgh & 0,273 & defg & 1,082 & fgh \\
\hline UFRGS 910906 & -- & & -- & & 1,204 & ijkl & 0,179 & efg & 0,394 & $\mathrm{i}$ \\
\hline UFRGS 960845-2 & -- & & --- & & 0,005 & $\mathrm{~m}$ & 0,026 & $\mathrm{~g}$ & 0,313 & $\mathrm{i}$ \\
\hline C. V. (\%) & \multicolumn{2}{|c|}{31,76} & \multicolumn{2}{|c|}{29,96} & \multicolumn{2}{|c|}{1,94} & \multicolumn{2}{|c|}{19,29} & \multicolumn{2}{|c|}{6,90} \\
\hline
\end{tabular}

Os dados sofreram transformação do tipo arco seno da raiz quadrada de (x/100).

Médias seguidas por letras distintas diferem entre si pelo teste de Tukey ao nível de 5\% de probabilidade.

o mais importante, e está relacionada tanto com a ocorrência de epidemias de ferrugem da folha da aveia (Simons, 1985) quanto com a expressão de alguns genes de resistência (Browder, 1985; Islam \& Mayo, 1990; Broers et al. 1996). Em 1998, a temperatura, desde o início do mês de agosto, esteve alta para a época (em torno de $18^{\circ} \mathrm{C}$ ), favorecendo a ocorrência precoce da doença e/ou alterando a expressão da resistência. Nos demais anos, a ferrugem foi observada no final de agosto/ início de setembro, coincidindo com o período em que a temperatura começou a tornar-se mais elevada (acima de 16 $\left.{ }^{\circ} \mathrm{C}\right)$. Em 1998 e 2000 verificaram-se altos índices de pluviosidade no período de desenvolvimento da doença, e em 1997, embora a quantidade de chuva tenha sido pequena, houve um pico pluviométrico no meio deste período. Estas observações sugerem que a quantidade de chuva também parece ter tido influência sobre a severidade da epidemia, possivelmente por proporcionar um período maior de molhamento foliar. A umidade relativa do ar e a radiação solar global, embora estejam ligadas à ocorrência de chuvas, não parecem ter tido influência sobre o desenvolvimento da doença.

A análise do comportamento dos genótipos ao longo dos anos em que foram testados, baseada nas variáveis quantificadas e na intensidade da epidemia em cada ano, permitiu que os mesmos fossem classificados em quatro grupos: resistentes (UFRGS 960845-2 e UFRGS 910906); moderadamente resistentes (UFRGS 41698-22, UFRGS 41709-5, UFRGS 42085 10, UFRGS 41721-21, UFRGS 9531-33, UFRGS 16, UFRGS 93641, UFRGS 93576 e UFRGS 953304); moderadamente suscetíveis (UFRGS 940263-3, UFRGS 94638-B, UFRGS 940772, UFRGS 953285 e CTC 3 ) e suscetíveis (UFRGS 7, UFRGS 10, UFRGS 14, UFRGS 17, UFRGS 41699-3, UFRGS 953109, UFRGS 952403, UFRGS 952602, UFRGS 922003, UFRGS 921260, UFRGS 950120, UFRGS 952576-1/2, UFRGS 960286-1 e UFRGS 953167).

Os genótipos resistentes tiveram um comportamento 
Resistência quantitativa à ferrugem da folha em genótipos de aveia...

estável, apresentando ao longo dos anos, baixos valores de AACPD (Tabela 2), severidade final e taxa de progresso da doença baixas (Tabelas 3 e 4) quando comparadas aos demais genótipos. Os genótipos com reação de resistência moderada, apresentaram, ao longo dos anos, valores de AACPD geralmente baixos a médios, (Tabela 2) e, no ano de 1998, chegaram ao final do ciclo com alta severidade da doença. $\mathrm{O}$ mérito destes genótipos está no seu comportamento nos anos em que a epidemia foi severa (1998 e 2000). Alguns destes genótipos tiveram baixas taxas de progresso em 1998, e em 2000, todos eles apresentaram esta característica (Tabela 4). Em 2000 a severidade final nestes genótipos alcançou níveis médios, quando comparada à da cultivar UFRGS 7, que chegou ao final do ciclo com $93,5 \%$ de severidade de ferrugem (Tabela $3)$. Os genótipos moderadamente suscetíveis apresentaram valores de AACPD médios a baixos, em relação ao grupo, em 1998; médios em 1999 e médios a altos em 2000 (Tabela 2), e, embora alguns tenham apresentado baixa taxa de progresso em 1998 (Tabela 4), todos chegaram ao final do ciclo nos anos de 1998 e 2000 com altos níveis de doença (Tabela 3). Os genótipos classificados como suscetíveis, apresentaram valores de AACPD médios a altos (Tabela 2), tiveram as maiores taxas de progresso da doença em 1998 (e alguns também em 2000) (Tabela 4) e chegaram ao final do ciclo nos anos de 1998 e 2000 com altos níveis de doença (Tabela 3 ).

Os genótipos classificados como resistentes e moderadamente resistentes apresentam bons níveis de resistência quantitativa, a qual foi responsável por uma considerável redução na AACPD, quando comparados aos genótipos suscetíveis. Em relação à AACPD da cultivar suscetível UFRGS 7 (considerada como 100\%), houve, na linhagem UFRGS 960845-2, uma redução de $99 \%, 94 \%$ e $88 \%$ na AACPD, nos anos de 1998, 1999 e 2000, respectivamente. Na linhagem UFRGS 910906, a redução na AACPD nos respectivos anos foi de $55 \%, 86 \%$ e $72 \%$.

Analisando-se a genealogia dos genótipos estudados (Tabela 1), verifica-se que houve variação no comportamento de linhagens oriundas do mesmo cruzamento, as quais aparecem em grupos diferentes. As linhagens UFRGS 910906 (resistente), UFRGS 41709-5 e UFRGS 953304 (moderadamente resistentes), e UFRGS 953285 e UFRGS 940772 (moderadamente suscetíveis), por exemplo, são oriundas do cruzamento UFRGS 15 x UFRGS 881920. O mesmo ocorre com as linhagens UFRGS 960845-2 (resistente) e UFRGS 940263-3 (moderadamente suscetível), as quais se originaram do cruzamento UFRGS $884110 \mathrm{x}$ UFRGS 884021. Outros exemplos são as linhagens oriundas dos cruzamentos UFRGS 881920 x UFRGS 14, UFRGS 10 x UFRGS 8 e UFRGS 884087 x UFRGS 15, as quais também apresentaram reações de resistência diferentes e nem sempre foram classificadas no mesmo grupo.

A seleção de genótipos de aveia com resistência quantitativa, deverá ser baseada no comportamento dos mesmos no campo, através da quantificação da AACPD, conforme sugerem Briere et al. (1994). O estudo das curvas de progresso da doença auxiliará a identificação de genótipos com um progresso lento (slow rusting). Esta característica pode mostrar-se estável por longos períodos de tempo (Wilcoxson, 1981), e, embora não possa ser considerada como um diagnóstico de resistência durável, sabe-se que certas cultivares que a apresentam têm mostrado resistência durável, sendo estes genótipos de grande valor para a investigação das causas da durabilidade (Johnson, 1984).

As avaliações de campo deverão ser feitas no maior número de ambientes (anos e locais) em que for possível para o programa de melhoramento, pois a expressão estável da resistência quantitativa em uma grande variedade de locais, embora desejável e conveniente para os melhoristas, não é necessariamente característica de todas as ferrugens de cereais. A expressão da resistência à ferrugem da folha da cevada, por exemplo, parece ser independente do ambiente (Parlevliet, 1975; Parlevliet \& Van Ommeren, 1975; Parlevliet et al., 1980), o que não se verifica para a ferrugem da folha do trigo (Parlevliet, 1988). Sugere-se ainda que a seleção de genótipos com resistência quantitativa à ferrugem da folha da aveia envolva a quantificação de seus componentes, para minimizar a possibilidade de que a resistência observada a campo não seja reflexo da baixa freqüência de raças virulentas do patógeno.

\section{REFERÊNCIAS BIBLIOGRÁFICAS}

ALEMAYEHU, F. \& PARLEVLIET, J.E. Variation for resistance to Puccinia hordei in Ethiopian barley landraces. Euphytica 90:365370.1996.

BRIERE, S.C., KUSHALAPPA, A.C. \& MATHER, D.E. Screening for partial resistance to an isolate of crown rust (Puccinia coronata $\mathrm{f}$. sp. avenae) race 264 in oat cultivars and breeding lines. Canadian Journal of Plant Pathology 16:49-55.1994.

BROERS, L.H.M., CUESTA-SUBIAS, X. \& LOPEZ-ATILANO, R.M. Field assessment of quantitative resistance to yellow rust in ten spring bread cultivars. Euphytica 90:9-16.1996.

BROWDER, L.E. Parasite: host: environment specificity in the cereal rusts. Annual Review of Phytopathology 23:201-222.1985.

CAMARGO, M.N., KLAMT, E. \& KAUFFMAN, J.H. Classificação dos solos usada em levantamentos pedológicos no Brasil. Boletim Informativo da Sociedade Brasileira de Ciência do Solo 12:1133. 1987.

CRUZ, R.P. da, FEDERIZZI, L.C. \& MILACH, S.C.K. Severidade da ferrugem da folha e seus efeitos sobre caracteres da panícula de aveia. Pesquisa Agropecuária Brasileira 34:543-551. 1999.

DOEHLERT, D.C. \& McMULLEN, M.S. Genotypic and environmental effects on oat milling characteristics and groat hardness. Cereal Chemistry 77:148-154. 2000.

FEDERIZZI, L.C. \& STUTHMAN, D. Porque genes maiores para resistência à ferrugem da folha têm pouca durabilidade no Brasil. Resumos, 18 Reunião da Comissão Brasileira de Pesquisa de Aveia, Londrina, PR. 1998. pp.1-2.

HARDER, D.E. \& HABER, S. Oat Diseases and Pathologic Techniques. In: Marshall H.G. \& Sorrels, M.E. (Co-Ed.) Oat Science and Technology. Madison. Crop Science Society of America. 1992. pp.307-402.

ISLAM, M.R. \& MAYO, G.M.E. A compendium on host genes in flax conferring resistance to flax rust. Plant Breeding 104: 89-100.1990. 


\section{M.S. Chaves et al.}

JOHNSON, R.A critical analysis of durable resistance. Annual Review of Phytopathology 22:309-330.1984.

JOHNSON, R. \& WILCOXSON, R.D. Components of slow-rusting in barley infected with Puccinia hordei. Phytopathology 68:14701474.1978.

LEONARD, K.J. O que sabemos sobre a ferrugem da folha em aveia? Palestra proferida durante a XIX Reunião da Comissão Brasileira de Pesquisa de Aveia, realizada de 30 de março a 1 de abril de 1999, Porto Alegre, RS.

LUKE, H.H., BARNETT, R.D. \& CHAPMAN, W.H. Types of horizontal resistance of oats to crown rust. Plant Disease Reporter 59:332-334. 1975

MARTINELLI, J.A., FEDERIZZI, L.C. \& BENEDETTI, A.C. Redução no rendimento de grãos de aveia em função da severidade da ferrugem da folha. Summa Phytopathologica 40:116-118.1994.

MARTINELLI, J.A., CHAVES, M.S. \& FEDERIZZI, L.C. MILACH, S.C.K. \& ALMEIDA, J.L. Análise da virulência de alguns isolados de Puccinia coronata avenae no sul do Brasil. Resumos, $18^{\mathrm{a}}$ Reunião da Comissão Brasileira de Pesquisa de Aveia, Londrina, PR. 1998. pp.17-19.

MELLO, D., LEMOS, R.C. da \& ABRÃO, P.U.R. Levantamento em série dos solos do centro agronômico. Porto Alegre. Faculdade de Agronomia e Veterinária da UFRGS. Relatório Técnico Científico, 8. 1996. pp.7-155.

NELSON, R.R. Genetics of horizontal resistance to plant disease. Annual Review of Phytopathology 16:359-378. 1978.

PARLEVLIET, J.E. Partial resistance of barley to leaf rust, Puccinia hordei. I- Effect of cultivar and development stage on latent period. Euphytica 24:21-27. 1975.

PARLEVLIET, J.E. Components of resistance that reduce the rate of epidemic development. Annual Review of Phytopathology 17:203222. 1979.

PARLEVLIET, J.E. Resistance of the nonrace-specific type. In: Bushnell, W.R. \& Roelfs, A.P. (Eds.) The Cereal Rusts vol II: Diseases, distribution, epidemiology and control. New York. Academic Press. 1985. pp.501-525.

PARLEVLIET, J.E. Strategies for the utilization of partial resistance for the control of cereal rusts. In: Simmonds, N. W. \& Rajaram, S. (Eds.) Breeding Strategies for Resistance to the Rusts of Wheat. Mexico. CYMMIT. 1988. pp.48-62.

PARLEVLIET, J.E. \& VAN OMMEREN, A. Partial resistance of barley to leaf rust, Puccinia hordei. II- Relationship between field trials, micro plot tests and latent period Euphytica 24:293-303. 1975.

PARLEVLIET, J.E., LINDHOUT, W.H. \& VAN OMMEREN, A. \& KUIPER, H.J. Level of partial resistance to leaf rust, Puccinia hordei in West European barley and how to select for it. Euphytica 29:1-81.
1980.

PATAKI, J.K., TOIT, L.J. du \& REVILLA, P. \& TRACY, W.F. Reactions of open-pollinated sweet corn cultivars to Stewat's wilt, common rust, northern leaf blight, and southern leaf blight. Plant Disease 82:939-944. 1998.

PRIESTLEY, R.H. \& BAYLES, R.A. The contribution and value of resistant cultivars to disease control in cereals. In: Clifford, B. C. \& Lester, E. (Eds.) Control of plant diseases: Costs and benefits. Oxford: Blackwell Scientific Publications, 1988. p. 53-65.

REES, R.G., THOMPSON, J.P. \& MAYER, R. Slow rusting and tolerance to rusts in wheat. The progress and effects of epidemics of Puccinia graminis tritici in selected wheat cultivars. Australian Journal of Agricultural Research 30:403-419. 1979a.

REES, R.G., THOMPSON, J.P. \& MAYER, R. Slow rusting and tolerance to rusts in wheat. The progress and effects of epidemics of Puccinia recondita tritici in selected wheat cultivars. Australian Journal of Agricultural Research 30:421-432. 1979b.

SHANER, G. \& FINNEY, R.E. The effect of nitrogen fertilizationon the expression of slow mildewing resistance in Knox wheat. Phytopathology 67:1051-1056. 1977.

SIMONS, M. D. Crown Rust. In: Roelfs, A.P. \& Bushnell, W.R. (Eds.). The Cereal Rusts: Diseases, distribution, epidemiology and control. New York. Academic Press. 1985. pp.132-172.

SIMONS, M.D., BROWNING, J.A. \& FREY, K.J. Modification of tolerance of oats to crown rust induced by chemical mutagens. In: Induced mutations for disease resistance in crop plants. Vol. II, Proc. Res. Coord. Meet., Riso, Denmark. STI/PUB/633. IAEA. Vienna, Austria. 1983

STUBBS, R.W., PRESCOTT, J.M., SAARI, E.E. \& DUBIN, H.J. Manual de metodologia sobre las enfermedades de los cereales. México. CYMMIT. 1986.

WAHL, I., ANIKSTER, Y., MANISTERSKI, J. \& SEGAL, A. Evolution at the center of origin. In: Bushnell, W.R. \& Roelfs, A.P. (Eds.) The Cereal Rusts: Origins, specificity, structure and physiology. New York. Academic Press. 1984. pp.39-72.

WILCOXSON, R.D. Genetics of slow rusting in cereals. Phytopathology 71:989-992. 1981.

ZHANG, Z.J. Evidence of durable resistance in nine Chinese land races and one Italian cultivar of Triticum aestivum to Puccinia striiformis. European Journal of Plant Pathology 101:405-409. 1995.

ZIMMER, D.E., SCHAFER, J.F. \& PATTERSON, F.L. Mutations for virulence in Puccinia coronata. Phytopathology 53:171-176. 1963.

ZONTA, E.P. \& MACHADO, A. SANEST. Sistema de Análise Estatística para Microcomputadores. Pelotas. Universidade Federal de Pelotas. 1984. 